\title{
Restoring Connectivity in a Resource Constrained WSN
}

\author{
Yatish K. Joshi and Mohamed Younis \\ Dept. of Computer Science and Electrical Engineering \\ University of Maryland Baltimore County \\ Baltimore, MD 21250 \\ yjoshi1, younis@umbc.edu
}

\begin{abstract}
Wireless Sensor Networks (WSNs) in applications like battlefield surveillance or environmental monitoring are usually deployed in inhospitable environments, in which their constituent nodes are susceptible to an increased risk of failure due to hazardous operating conditions or adversary attacks. In these scenarios it is possible for multiple nodes to fail at the same time and partition the WSN into disjoint segments. Such loss of connectivity may cause service disruptions and render the WSN useless. Given the critical role a WSN plays and the fact that deployment of additional nodes may be infeasible, the WSN must have the ability to self-heal and restore connectivity by utilizing surviving resources. In this paper we present a distributed

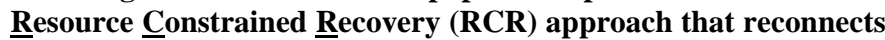
a network partitioned into disjoint segments by strategically repositioning nodes to act as relays. In case the number of surviving relocatable nodes are insufficient to form a stable intersegment topology, some of them are employed as mobile data collectors with optimized tours to reduce data latency. The performance of $\mathrm{RCR}$ is validated through mathematical analysis and simulation.
\end{abstract}

Keywords: Topology repair, Fault recovery, Connectivity restoration, Fault-tolerance, Wireless sensor networks, Mobile Data Collectors.

\section{INTRODUCTION}

Due to their cost advantage, ease of use, rapid deployment and round the clock operation, the use of Wireless Sensor Networks (WSN) is increasingly prevalent in applications designed to operate in harsh environments like battlefields, border surveillance, rain forests etc. In these applications, the WSN is setup in an ad-hoc manner, with sensor nodes being deployed aerially in the area of interest, and once on the ground coordinating with one another to form an interconnected network to carry out application specific tasks. In such setup and operation scenarios nodes have an increased risk of failure due to the harsh environmental conditions or due to enemy action. For example, bombing or missile strikes in a battlefield or a forest fire could render a large collection of nodes inoperable and partition the network into disjoint segments. Given the unattended operation and the infeasibility of timely deployment of additional nodes, the WSN must be able to recover from collocated node failures autonomously in order to restore connectivity and resume its service.

Multiple strategies have been pursued to tolerate multi node failures in WSNs [1]. Published schemes can be classified broadly into proactive and reactive strategies. Proactive strategies involve careful node-placement wherein node positions are determined prior to network deployment and then leveraged to provision redundancy by forming a kconnected topology [2][3][4] or using redundant nodes as backups [5][6]. Although a proactive strategy can lower the risk of network partitioning, it does not provide sufficient mitigation in ad-hoc network formation scenarios since the node positions cannot be accurately determined at time of deployment and a large scale failure may damage some nodes and all their backups.

Reactive strategies on the other hand are based on reconfiguring the network topology after failure. Basically, an inter-segment topology is formed in order to reestablish connectivity amongst disjoint segments. Reactive strategies can be broken down into two main classes, namely, centralized and distributed, based on the amount of information available during the recovery process. A centralized approach assumes the knowledge of the entire network state, which is exploited to find an optimal recovery solution. The recovery basically boils down to a node placement problem for which there are a number of published heuristics [8][9][10][11]. Meanwhile, distributed approaches rely on local state information and try to reconnect the network by either having representative nodes from the segments meet at a common point [12][13][14] or exploiting the shape of the network topology before failure to determine the recovery paths [15][16]. Although centralized approaches yield optimized solutions, they require external resources, i.e., aerial support from satellites, aircrafts or UAVs to collect and disseminate global network state information on demand. Such external support may not be available at all times or be feasible due to budgetary constraints. Therefore, distributed approaches are deemed more practical for ad-hoc formed WSNs.

Contribution: In this paper we study the connectivity restoration problem under resource availability constraints. The problem is motivated by the fact that after a catastrophic failure the surviving segments may not have enough mobile nodes that can serve as relay nodes (RNs) and form a stable inter-segment topology. Basically, most distributed recovery approaches found in the literature, e.g., [13][14][15][16], are based on the assumption that the surviving segments have sufficient RNs within them and these RNs are available for repositioning without negatively affecting the intra-segment connectivity. This assumption, however, may not hold in practical scenarios where failures randomly take place and a segment may have insufficient RN count to support recovery. To tackle such a challenging recovery problem, we present a novel Resource Constrained $\underline{R}$ ecovery (RCR) approach.

RCR aims to reconnect the disjoint segments in a partitioned WSN that has a fixed number ' $l$ ' of available RNs that is insufficient for forming a stable inter-segment topology. Therefore, RCR utilizes the available RNs to provide intermittent connectivity amongst the segments. We make the recovery problem even more realistic by restricting the capability of the available RNs. Out of the ' $l$ ' RNs available we consider ' $l_{S}$ ' of them to be stationary RNs and ' $l_{M}$ ' to be mobile RNs that can be utilized as mobile data collectors (MDCs). This restriction is due to the fact that some RNs may have low battery life either due to the overhead experienced 


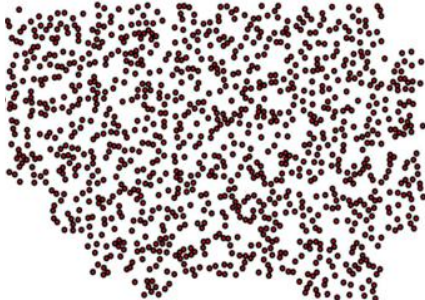

(a)

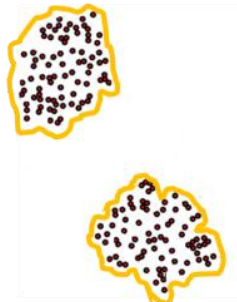

(b)

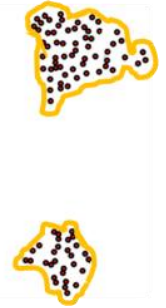

Figure 1: Overview of RCR. (a) An ad-hoc WSN pre-failure, (b) WSN partitioned into 4 disjoint segments after a catastrophic failure (c)

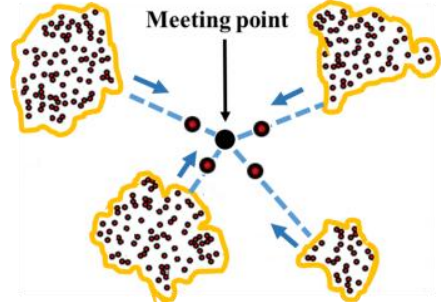

(c)

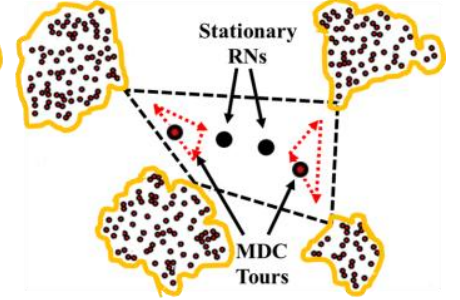

(d) Segments populating RNs towards a common meeting point and discovering one another (d) Two RNs employed as stationary relay nodes to shorten the MDC tours.

while participating in the recovery process, or because they may have suffered some damage that impairs their movement. Therefore it is practical for these RNs to be stationary and act as an interface between disjoint segments in order to prolong their lifetime.

Figure 1 gives a succinct overview of our strategy. Given an ad-hoc WSN, as seen in Figure 1(a), that suffered a large scale failure due to an external event, the network is partitioned into four disjoint segments as in Figure 1(b). Our aim is for the disjoint network segments to discover one another and reestablish communication links between them by employing the surviving relay nodes. In the first phase highlighted by Figure 1(c), segments populate representative RNs towards a common meeting point, the location of which is determined prior to failure and stored within network nodes. If the segment has excess RNs to spare for recovery, they follow the leading $\mathrm{RN}$ in a cascaded manner towards the meeting point. Once connected at the center, the RNs exchange information with one another and now know the number of RNs available for recovery and how many of them can be employed as MDCs and stationary RNs. Based on this information exchange, in the second phase the segments are divided into groups whose count is equal to the number of MDCs so that each MDC is assigned a subset of segments to tour. In the segment grouping process, we can utilize some of the stationary RNs as interfaces between various groups to ensure balanced tour loads on the MDCs. In the third and final phase the remaining stationary RNs that are unutilized during the grouping process are employed to shorten the travel path of the largest MDC tours. Figure 1(d) shows the final reconnected topology.

$\mathrm{RCR}$ is validated through simulation experiments. The simulation results show that RCR outperforms competing schemes and produces tours that are not only smaller in total length but they also share the travel load more equitably and thus improve data latency. The rest of the paper is organized as follows. The next section sets RCR apart from existing solutions. Section III discusses the system model. Section IV describes RCR in detail. Section V reports the simulation results. Section VI concludes the paper.

\section{RELATED WORK}

As pointed out in the previous section, proactive strategies that aim to exploit redundancy as a recovery mechanism by forming k-connected topologies do not scale well to handle multi-node failures. In addition, distributed reactive recovery solutions that deal with single node failures e.g., [17][18][19], cannot be scaled to handle multi-node failures. These solutions require the neighbors of a failed node to collaborate with one another, to either find a replacement for the failed node, or inward movement by all neighbors until connectivity is reestablished. This reliance on neighbors of a failed node does not scale for multi node failures, since in a multi node failure scenario, the scope of failure is unknown, i.e. the nearest healthy node may be many hops away. Surviving nodes will not know in which direction to proceed for recovery unless they store multi-hop information and maintaining a global view of the network state imposes significant messaging and storage overhead.

In the remainder of this section we set RCR apart from published recovery solutions that tackle multi-node failures.

Centralized approaches such as FESTA [10] and IO-DT [11] treat recovery as a relay placement problem, which is equivalent to solving for the Steiner Minimum Tree with Minimal Steiner Points (SPs) and Bounded Edge-Length (SMT-MSPBEL) shown to be NP-Hard by Lin and Xue [7]. The solution provides the minimum number and position of RNs that need to be deployed in order to reconnect the network. Although these approaches provide the best possible solution for recovery, they require the entire network state. Hence their use may not be possible in a resource constrained ad-hoc network that does not have access to satellite links or airborne units to provide the entire state. Also these centralized approaches cannot tackle recovery when the number of surviving RNs in the damaged WSN is less than the number of SPs.

On the other hand, distributed recovery approaches rely only on the local state, obviously at the expense of optimality. For example, AuR [12] utilizes 1-hop information to perform a combination of self-spreading and movement inwards toward the center of the deployment area to guarantee convergence and reconnect the disjoint segments in a partitioned network. Although AuR can handle the resourceconstrained recovery problem that RCR aims to tackle, AuR requires all nodes in the WSN to be mobile and thus may not be applicable in all WSN applications wherein a network can be composed of a of a mixture of stationary sensor nodes and mobile relays.

DORMS [13] and DarDs [14] utilize mobile RNs to restore connectivity. The basic idea is to place RNs towards a pre-determined common point in the deployment area along the shortest path and form an inter-segment topology that restores connectivity. Once initial connectivity is restored an optimization phase is run to reduce the number of deployed RNs. SSBR [14] and GSR [15] pursue a different approach; instead of having a common meeting point, they exploit the pre-failure topology of the network to determine recovery paths that can be populated with RNs in case of failure. While highly effective, they along with DORMS and DarDs operate 
under the assumption that the disjoint segments collectively have sufficient RNs to form a stable inter-segment topology and do not consider the case where the number of available RNs are insufficient for forming a stable inter-segment topology.

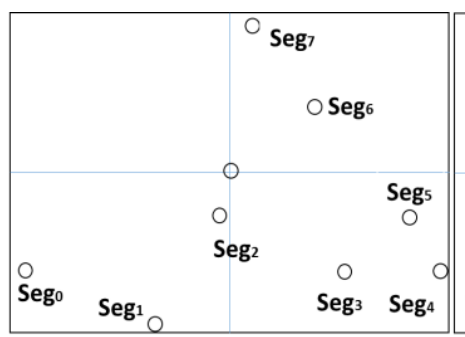

(a)

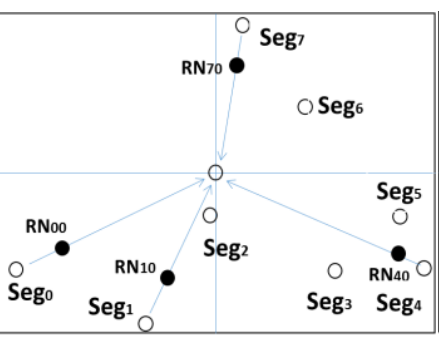

(b) groups that can be toured by a MDC and utilizes stationary RNs to shorten the longest MDC travel path. RCR also deals with recovery under the same constraint as MiMSI. However, unlike other approaches, it not only aims to minimize the total MDC tour length but also focuses on creating balanced MDC

Figure 2: Initial Relay Deployment Phase (a) Eight surviving segments in a damaged WSN, (b) RN positions after some time T, black circles represent deployed RNs (c) : Position after time $T+\Omega$.(d) Center connected topology achieved.

Mobile data collectors (MDCs) or data mules have been used for data collection and dissemination in sparse or fragmented networks wherein direct communication links are absent between different network components [20][21][22] [23]. The idea of using MDCs as a medium to provide intermittent connectivity presented in these schemes can be utilized in a damaged WSN to restore connectivity amongst disjoint segments when a stable topology cannot be formed due to limited $\mathrm{RN}$ availability. Approaches like [24][25] handle recovery by focusing on data delivery. In [24], a data delivery model utilizing MDCs is presented; it basically considers the effect of the number of available MDCs on the end-to-end delay. Meanwhile in [25], a MDC tour is designed to minimize data loss rates and looks at the data generation frequency in determining tour paths. However, these approaches are centralized in nature and require the location of segments and scope of failure to be determined by external means. Moreover, they do not consider MDC tour length as a metric.

On the other hand, some MDC-based recovery solutions try to find the shortest MDC tour that covers the segments under each MDC's purview. This reduction in tour not only improves data latency but saves energy, thus extending a MDC's lifetime. Finding the shortest tour though is a traveling salesman problem, which is known to be NP-hard [33] hence the recovery solutions pursue polynomial time heuristics to determine a MDC's tour path. Approaches like MINDS [29] and IDM-kMDC [30] divide the set of disjoint segments into groups; each is toured by a MDC. Both MINDS and IDMkMDC form a minimum spanning tree (mst) over the set of disjoint segments and utilize it as the backbone to divide segments into groups. MINDS [29] divides the mst around its center and recursively keeps dividing the larger half until there is a group for every available MDC. IDM-kMDC [30] on the other hand pursues a greedy approach; it initially assigns a MDC to every mst edge and then continuously merges the two MDC tours that have the least increase in overall tour length until the number of tours equals the number of available MDCs. However, none of these approaches consider the case where some of the available RNs cannot take part in touring and have to be stationary in the repaired topology.

MiMSI [31] deals with a more constrained version of the problem wherein not all available $\mathrm{RNs}$ can be utilized as MDCs and some may have to be utilized as stationary RNs. MiMSI uses kmeans++ [32] to divide disjoint segments into tours to ensure that no single MDC is overloaded and thus strives improve data latency.

We compare the performance RCR with MiMSI and show that our approach outperforms MiMSI in all relevant metrics such as total MDC tour length, the maximum touring load on a MDC and the standard deviation amongst all MDC tours.

\section{SYSTEM MODEL}

The proposed RCR approach considers an ad-hoc WSN composed of a mix of stationary sensor nodes and mobile relay nodes (RNs) or all RNs that has been partitioned into multiple disjoint segments due to the failure of multiple collocated nodes. We assume that a $\mathrm{RN}$ is aware of the size of the deployment area and its position, e.g., using contemporary localization schemes. Prior to failure, the network determines a common meeting point e.g., center of deployment area, which is stored by RNs and is utilized during the recovery process to link up with other survivors. All RNs are assumed to have the same communication range $\mathrm{R}$. The surviving nodes at the periphery of failure detect node failures by assessing the number of neighbors they have lost contact with and inability to reach other parts of the network, etc. [27]. The survivors group themselves into a connected segment and act as one entity for recovery.

Each segment picks a $\mathrm{RN}$ to lead the recovery process and is followed by other RNs if available. The selection of the leader can simply be based on its proximity to the common meeting point. Figure 2(a) shows a WSN that has been partitioned into eight disjoint segments $\mathrm{Seg}_{0}, \mathrm{Seg}_{1}, \ldots, \mathrm{Seg}_{7}$ due to multi-node failure. The center of the deployment area ' $O$ ' is considered the common meeting point for the remainder of the discussion. RCR aims to connect these disjoint segments using the surviving RNs.

\section{RCR APPROACH}

RCR consists of three phases, namely, initial relay deployment, segment grouping phase, and a tour optimization phase. These phases are described in detail in the balance of this section.

\section{A. Initial Relay Deployment}

After a network has been partitioned into multiple disjoint segments, the surviving segments do not have any knowledge about each other's position. So to restore initial connectivity and get in contact with other survivors, each segment 
populates RNs towards a pre-determined common point decided before failure, e.g., the center of the deployment area ' $O$ '. It is assumed that each segment has at least one RN available for recovery purposes, otherwise it will remain unconnected unless it lies in the travel path of another segment's RN. The relocation is led by the leading RN of each segment as shown in Figure 2(b). To ensure coordination during redeployment and to associate $\mathrm{RNs}$ to their respective segments, the relays are uniquely labeled as $R N_{i j}$, where ' $i$ ' is the segment ID which identifies the association of relay and ' $j$ ' is the index which distinguishes the order of relays deployed in the path from the segment to ' $O$ '. The leading relay is identified as $R N_{i 0}$. The number of RNs required to reach any node at ' $O$ ' from a segment ' $i$ ' is:

$$
N_{\text {Relays }}=\frac{\operatorname{Dist}\left(\operatorname{Seg}_{i}, O\right)}{R}-1
$$

Where $\operatorname{Dist}\left(\operatorname{Seg}_{i}, O\right)$ is the Euclidean distance between the segment and ' $O$ '.

The farthest segment from the center has to be located on the diagonal of the deployment area and be half the diagonal length away. The maximum time that can be taken by a RN from the farthest segment to reach the center ' $O$ 'is given by:

$$
T_{\text {Max_center }}=\frac{L_{\text {Diagonal }}}{2 v}
$$

Where $L_{\text {Diagonal }}$ is the diagonal of the deployment area and ' $v$ ' is the average speed of a RN. Therefore for any segment $\mathrm{Seg}_{i}$ located in the interior of the deployment area, the time to reach the center ' $O$ ' is given by:

$$
T_{\text {to_center }}(\mathrm{i})=\frac{\text { Distance to center }}{v}
$$

All segments may not have $N_{\text {Relays }}$ available RNs. Therefore to ensure proper utilization of the deployed RNs, each segment $\mathrm{Seg}_{i}$ waits for $T_{\text {Wait }}(i)$ amount of time for leading RNs of segments farthest from ' $O$ ' to come with contact range of the nodes of $\mathrm{Seg}_{i}$, before $\mathrm{Seg}_{i}$ decides to sends RNs towards ' $O$ ', where:

$$
T_{\text {wait }}(i)=T_{\text {Max_center }}-T_{\text {to_center }}(i)
$$

This wait time ensures that an interior segment will not unnecessarily deploy RNs if it lies on the path of outer segments towards ' $O$ ', and thus saving the energy of its RNs. $\mathrm{RCR}$ aims to minimize the number of RNs deployed to reach the center but also reduce the $\mathrm{RN}$ travel distance; therefore $T_{\text {Wait }}$ allows segments to discover one another before reaching ' $O$ '. The inward motion allows multiple leading RNs to get in range of one another on route to the center, where they exchange their respective segment information and the closest $\mathrm{RN}$ to the center amongst them continues until reaching ' $O$ ' while the others stop.

For example in Figure 2(a), each segment has just one mobile RN available for recovery. After a failure, $\mathrm{Seg}_{0}, \mathrm{Seg}_{1}$, $\mathrm{Seg}_{4}, \mathrm{Seg}_{7}$ deploy their only RN towards ' $O$ ' before other segments, as seen in Figure 2(b). This is due to the fact that they lie on the periphery of the WSN and hence have a smaller $T_{\text {wait }}$ time compared to interior segments. As seen in Figure 2(b), during the inward motion towards the center, $R N_{40}$ from $\mathrm{Seg}_{4}$ comes within the communication range of nodes in $\mathrm{Seg}_{5}$ and stops. After $\mathrm{Seg}_{5}$ 's $T_{\text {wait }}$ is over, its leading $R N_{50}$ is deployed towards the center as seen in Figure 2(c); such a leading $\mathrm{RN}$ also has information regarding the position of $R N_{40}$ and $\mathrm{Seg}_{4}$ that will be utilized in later phases. As seen in
Figure 2(d), the leading RNs of $\mathrm{Seg}_{6}$ and $\mathrm{Seg}_{7}, \mathrm{Seg}_{3}$ and $\mathrm{Seg}_{5}$ come in the communication range of one another and the closest $\mathrm{RN}$ to ' $O$ ' obtains the segment information from the other RNs, and continue toward the center. Similarly $R N_{10}$ of $\mathrm{Seg}_{1}$ comes in contact with $\mathrm{Seg}_{2}$ and stops. Given the close proximity of $\mathrm{Seg}_{2}$ to ' $O$ ', it has the largest $T_{\text {wait }}$ and is the last to be deployed and proceeds to reach ' $\underline{O}$ '. $R N_{00}, R N_{30}$ and $R N_{60}$ of the other segments stop when they come within its communication range of $R N_{20}$, resulting in a center connected topology as seen in Figure 2(d).

The maximum $\mathrm{RN}$ count required to establish a connected inter-segment topology will be less than or equal to the sum of $R N_{O}$ and all RNs on the paths from the individual segments toward ' $O$ ' until becoming in range of $R N_{O}$. Thus, the maximum number of RNs that can be deployed during initial relay deployment can be given by:

$$
\operatorname{Max}_{\text {Relays }} \leq \sum_{i=1}^{\# \text { segments }} N_{\text {Relays }}+1
$$

Note that in practice the actual RN count is often less than $\operatorname{Max}_{\text {Relays }}$ since some of the RN paths from the individual segments get merged during the inward relay deployment. Since we are considering a resource constrained WSN, the number of RNs available for connectivity will always be less than Max $_{\text {Relays. }}$ For example in the WSN depicted in Figure 2 the number of relays needed to establish a stable inter-segment topology without taking merging into account is 20 , although it only has 8 RNs available for recovery.

Theorem 1: RCR converges to an initial connected topology in constant time.

Proof: In the initial phase, RNs are populated by all surviving segments until a leading $\mathrm{RN}$ becomes reachable to the center, i.e., becomes $R$ units away from ' $O$ ', or merges with a segment while on route to ' $O$ '. Only one of these leading RNs will eventually be positioned at ' $O$ '. In the worst case scenario the surviving segments can be located at the corners of a rectangular deployment area, so the maximum distance between a segment and the center will be half the diagonal length. Assuming at least one segment is located at the corners of the deployment area and travel inwards at a speed ' $v$ ', the time taken will be given by distance divided by speed, i.e., $\left(\frac{L_{\text {Diagonal }} / 2}{v}\right)$, which is a constant. $\square$

Lemma 1: There can only be a maximum of six segments that do not merge before reaching the center.

Proof: For segment representatives to meet at the center, they need to be separated by ' $R$ ' when they come in contact with $R N_{O}$. As shown in Figure 3, only six such positions can exist since if a pair of adjacent segments are separated by ' $R$ ' and equidistant from the center, they form an equilateral triangle which has a center angle of 60 degrees. The center subtends an angle of 360 degrees; therefore there exist only 6 segments that will meet while being ' $R$ ' away from the center. Thus, for $N_{\text {Seg }}>6$, segments will merge before reaching the center and the number of employed RNs and the total distance they have to travel in the initial phase will be reduced significantly.

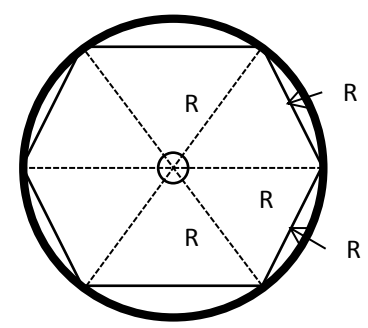

Figure 3: The topology at the center with segments separated from each other and the center by $\mathrm{R}$. 


\section{B. Segment Grouping}

After the initial relay deployment phase, the leading RNs of different segments are connected with one another around ' $O$ '. $R N_{O}$ would then know the position of all disjoint segments and the resources available within them for recovery. $R N_{O}$ divides the available RNs into $l_{S}$ stationary RNs and $l_{M}$ RNs to be utilized as MDCs based on a pre-determined user criterion that could vary according to the application and deployment scenario and could also factor in the remaining energy level of the $\mathrm{RN}$, or the relay's condition, i.e., physical damage that may constrain movement. In order to establish inter-segment connectivity, RCR forms $l_{M}$ groups of segments; the segments of each group is to be toured by a MDC. The objectives for segment grouping are: (i) the tour length of individual MDCs is to be balanced in order to reduce the maximum data delivery delay and equalize the motion overhead, (ii) simplify the inter-segment data forwarding by relaying data among MDCs through segments, and (iii) minimize the travel overhead on individual MDCs.

To achieve the above objectives, RCR first picks a terminal, i.e., one of its nodes, $S_{i}$ for each segment $S e g_{\mathrm{i}}$ to act the segment's interface with the MDC for date exchange. Thus, the aim of segment grouping becomes to identify terminals that can serve as rendezvous points between $l_{M}$ groups such that MDC tour length for each group is nearly equal. This ensures that the load on the MDCs is balanced and reduces the data delivery latency. Popular proximity-based clustering approaches like kmeans++ focus on minimizing the distance between the group members, i.e., minimize the intracluster tour, and do not factor in inter-cluster connectivity as a design goal. In other words, after clusters are formed, these approaches identify terminals that can serve as gateways for data exchange between the clusters, which often results in poor choice of rendezvous points. Unlike these proximitybased clustering approaches, RCR determines the gateway terminals around which clusters can be built such that the clusters formed have balanced tours.

To identify the gateway terminal between segments groups RCR employs the concept of eccentricity of a graph [35]. Given a graph $G(V, E)$ that consists of the set of vertices $V$ and the set of edges $E$, the eccentricity of a vertex $v \in V$ is defined as the distance from $v$ to the most distant vertex. A vertex of minimum eccentricity is called the center of a graph. By modeling the terminals as a graph, RCR opts to employ the center of such a graph as a rendezvous terminal in order to minimize the maximum data delivery delay, i.e., achieving objective (i) above. Having a rendezvous terminal that is part of the tour of two MDCs obviously simplifies data exchange between them and thus achieves objective (ii). RCR pursues two methods for factoring in the distance $d(x, y)$ between two vertices $x$ and $y$, namely, (1) $d(x, y)$ is the length of the shortest multi-hop path between $x$ and $y$, and (2) $d(x, y)$ is the length of the edge between $x$ and $y$. The former is intuitive since all terminals that are to be connected by an MDC, which will have to hop amongst them in its tour. Since RCR opts to minimize the travel overhead, i.e., objective (iii) above, we consider the minimum spanning tree ( $m s t$ ) of the terminals as the underlying terminal graph in this case. The cost of constructing an $m s t$ is $O(|E| \log |V|)$. In summary, RCR considers the following two scenarios:
1. What is the optimal rendezvous terminal if we consider only mst edges, i.e., if a path connecting $x$ and $y$ does not exist we set $d(x, y)=\infty$.

2. What is the optimal rendezvous terminal if we assume there exists an edge connecting every pair of vertices.

These two scenarios can be recast as optimization problems based on the distance matrix $D=(d(x, y))$ of $G$. The eccentricity $e(x)$ of a vertex $x$ in $G$ and the radius of a graph $\rho(G)$ is defined as:

$$
\begin{aligned}
& e(x)=\max _{y \in V} d(x, y) \\
& \rho(G)=\min _{x \in V} e(x)
\end{aligned}
$$

The center of $G$ is the set of vertices that have the minimum eccentricity, i.e.,

$$
C(G)=\{x \in V \mid e(x)=\rho(G)\}
$$

So we solve for the graph center in both scenarios; one where we consider only mst edges in $D_{l}=(d(x, y))$, and in the other we consider an edge existing between every pair of vertices $D_{2}=(d(x, y))$. We find candidates for the graph center in both these scenarios and qualify the best choice based on the tours that could be formed when a candidate vertex is picked as a rendezvous terminal. Basically, we utilize the mst as the underlying structure to divide the set of terminals around the candidate vertex [29] and determine the tour lengths $T_{1}$ and $T_{2}$ in each case. The candidate vertex which results in the least difference in tour lengths $\left(T_{1}-T_{2}\right)$ is chosen as the rendezvous terminal.

The question that arises is why RCR considers two scenarios to select the best rendezvous terminal. The reason is because of the vagaries of the $m s t$, which is highly dependent on the segment positions; sometimes just considering the mst edges in the distance matrix $D=(d(x, y))$ of $G$ results in a nonideal choice. This is illustrated in Figure 4(a), which shows four terminals $S_{0}, S_{1}, S_{2}$ and $S_{3}$ representing their respective

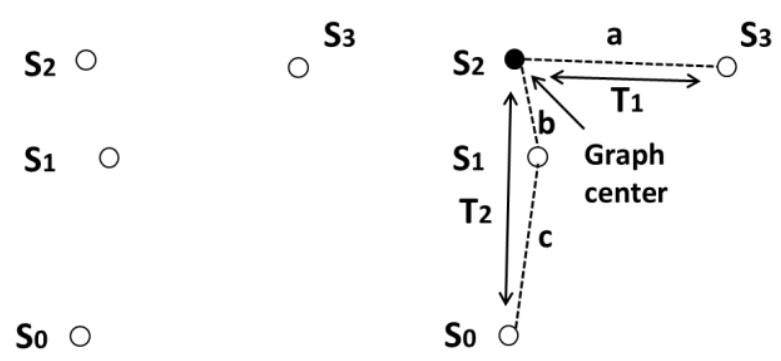

(a)

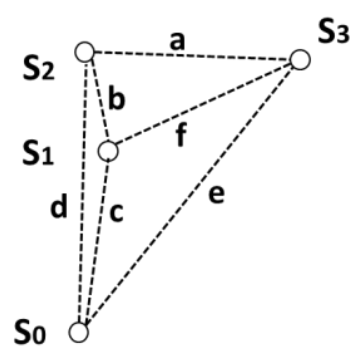

(c) (b)

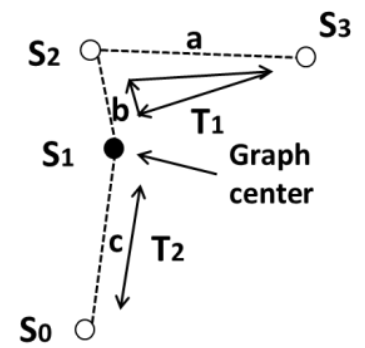

(d)
Figure 4: Illustrating the selection of graph centers for segment grouping; (a) An example topology with four terminals $S_{0}, S_{1}, S_{2}$ and $S_{3}$ representing their respective segments $S_{e g}, S_{1} g_{1} S_{2} g_{2}$ and $\mathrm{Seg}_{3}$; (b) Scenario \#1 where only $m s t$ edges are considered in the distance matrix, results in identifying $\mathrm{S}_{2}$ as the graph center; (c) Scenario \#2 where we consider an edge to exist between every pair of vertices; (d) The graph center for scenario \#2 is located at 
segments $\mathrm{Seg}_{0}, \mathrm{Seg}_{1}, \mathrm{Seg}_{2}$ and $\mathrm{Seg}_{3}$. For these segments the rendezvous terminal is to be determined. As seen in Figure 4(b), the mst edges connecting the terminals are of length $a, b$ and $c$, respectively with $(b<c<a)$. When using only mst edges in $D_{l}=(d(x, y))$, we end up with $S_{2}$ as the graph center since $(b+c)<(a+b)$ and thus $S_{2}$ has the minimum eccentricity, i.e., $d\left(S_{2}, S_{0}\right)<d\left(S_{1}, S_{3}\right)$. Meanwhile in the second scenario depicted in Figure 4(c) and 4(d), we assume that an edge exists between every pair of vertices, which leads to identifying $S_{1}$ as the graph center. The resulting tours show that not only is the tour length more equitable if $S_{1}$ is used as a rendezvous terminal for this topology, but the overall tour length is also shorter. Thus, by choosing the graph center that results in the most balanced division, we obtain two nearly equal sized groups.

The way RCR performs segment grouping differs based on the availability of $l_{S}$ RNs as follows:

(1) $\underline{l}_{\underline{S}}=0$ : If all RNs can be utilized as MDCs, we simply determine the graph center and divide the mst around it. We recursively keep dividing the larger of the resulting parts, i.e., find the graph center of that part, until we obtain $l_{M}$ groups.

(2) $\underline{l}_{s} \geq 0$ : If some of the RNs have be stationary, RCR utilizes them to find a graph center around which a more equitable group sizes can be obtained. The question is where to place the stationary RNs so as to improve segment grouping. To answer this question, RCR models the recovery problem as RN placement problem which is equivalent to finding the Steiner Minimum Tree with Minimal Steiner Points and Bounded Edge-Length (SMT-MSPBEL). SMT-MSPBEL is an NP-hard problem for which numerous heuristics have been proposed. Among published solutions, RCR employs IO-DT [11] which is one the latest and most effective heuristics. The Steiner points (SPs) determined by IO-DT help in pointing out potential positions where stationary RNs can be deployed to act as gateways between segments. Unlike the case of $l_{s}=0, \mathrm{RCR}$ considers the SPs, identified by IO-DT, along with the segment terminals as vertices in $G$. We determine the graph center and if it lies on a SP we mark it as a place to deploy a stationary $\mathrm{RN}$ and decrement the $l_{S}$ count. This process continues until we obtain $l_{M}$ groups. In case if we run out of stationary RNs before obtaining $l_{M}$ groups, the unused SPs are removed from $G$ and RCR proceeds as in the case of $l_{S}=0$, above. As will be shown in the Section $\mathrm{V}$, our approach yields finer groupings because by having more vertices in the $m s t$, we have shorter edges in $G$. The grouping process will be illustrated through a detailed example later in this section.

Lemma 2: If $l_{S}>0$, RCR forms an SMT-MSPBEL utilizing the IO-DT heuristic whose time complexity is $O\left(n^{2}\right)$ [11].

Lemma 3: In an mst, the graph center can be determined in $O(n)$.

Proof: The longest path in an mst can be found by running breadth-first search (BFS) rooted at any vertex say $v_{i}$ in the $m s t$ and finding the farthest vertex $v_{k}$, and then doing the same for $v_{k}$ to determine the farthest vertex from it in the mst. The time complexity of these steps is $O(E)$ or $O(n-1)$ as there are $n-1$ mst edges over $n$ vertices. The graph center is located at the vertex whose distance to the farthest vertices of the longest path is the smallest. The longest path can comprise of at most $n-2$ vertices and $n-3 m s t$ edges. Thus, finding the center of the mst is $O(n)$.

Lemma 4: In a graph $G$ where an edge exists between every pair of vertices the graph center can be determined in $O\left(n^{2}\right)$.

Proof: In an mst comprising of $n$ vertices, in order to find the longest edge each vertex needs to check its distance to the remaining $(n-1)$ vertices, doing this for every vertex leads to a time complexity of $O\left(n^{2}\right)$.

Theorem 2: The runtime complexity of determining all graph centers in the segment grouping phase is $O\left(\left(l_{M}-1\right) n^{2}\right)$.

Proof: Most of the work in this phase is done in recursively splitting the segments until we obtain $l_{M}$ segment groups. This takes at most $\left(l_{M}-1\right)$ iterations. For each iteration it takes $O(n)$ and $O\left(n^{2}\right)$ time to find the graph center, according to Lemmas 3 and 4 above. Therefore, the runtime complexity of one iteration is $O\left(n^{2}\right)$ and for $\left(l_{M}-1\right)$ iterations the overall time complexity will be $O\left(\left(l_{M}-1\right) n^{2}\right)$.

\section{Tour Formation}

At the end of the segment grouping phase, we obtain $l_{M}$ groups each with its own assigned MDC. In this subsection we explain how the MDC will tour the segments in its assigned group. Each segment $S e g_{\mathrm{i}}$ selects a terminal $S_{\mathrm{i}}$ to act as the segment's interface with the MDC for date exchange. An MDC tour $T_{s}$ is defined as the shortest possible cycle that visits every terminal $S_{0}, S_{1}, S_{2}, S_{3 \ldots .} S_{\mathrm{q}}$ in its list for data collection and dissemination and returns to its starting position. Finding the shortest possible MDC tour is equivalent to the Euclidean Travelling Salesman Problem and is NP-Hard [33]. Also for data exchange to take place between a MDC and a terminal, the MDC must pass within the transmission range of the terminal, we call these data exchange stops as collections points. This section describes how RCR forms the smallest MDC tour and determines the collection points for the MDC. The tour formation can be divided into two cases based on the number of terminals that need to be covered by the MDC.

- No of Terminals =2: This is the simplest case to handle, here an MDC is used to provide connectivity between two terminals. The shortest path between two points is a straight line connecting them. If the distance ' $\delta$ ' between two points is $R<\delta \leq 2 R$, where $R$ is the communication range, the MDC will be stationary and positioned at the midpoint of the line connecting the two terminals, as seen in Figure 5(a). If $\delta>2 R$, the collection points for data exchange are located at the intersection of the transmission discs of terminals $S_{l}$

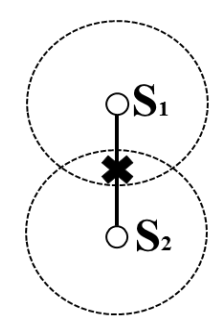

(a)

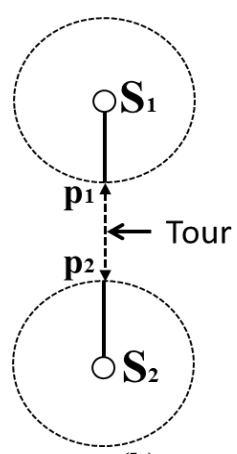

(b)
Figure 5: Determining the tour between two terminals when the distance between them is, (a) $R<\delta \leq 2 R$ and (b) $\delta>2 R$ 
and $S_{2}$ of radius $R$ and the line connecting them. As seen in Figure 5(b), the MDC will move back and forth between collection points $P_{1}$ and $P_{2}$ for data exchange.

- No of Terminals >2: In case a MDC has to tour more than two terminals, RCR forms a simple polygon where all terminals are located on its boundary. To do so RCR first constructs a convex hull over the terminals and then includes interior terminals by replacing their closest edges on the convex hull. To illustrate, we consider the example in Figure 6(a), where seven terminals are to be toured by an MDC. As seen in Figure 6(b), we determine the convex hull, resulting in 5 convex and 2 interior terminals. For each interior terminal, RCR finds the closest convex edge as shown in Figure 6(c) and then that edge is adjusted to include the interior terminal as well, resulting in a simple polygon depicted in Figure 6(d).

The simple polygon obtained gives us a MDC tour that covers all terminals, but we still need to determine the collection point for every terminal that an MDC need to stops at. For the shortest MDC tour, the collection points have to lie in the interior of the polygon. The following theorem provides a guideline for selecting the collection point for a terminal.

Theorem 3: The maximum reduction in the perimeter of a simple polygon of terminals is achieved when the collection

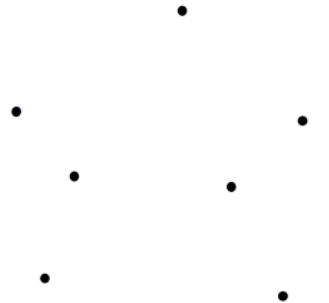

(a)

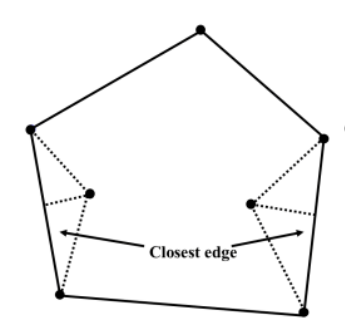

(c)

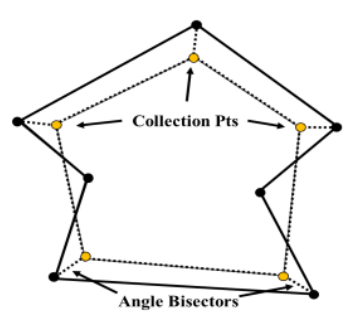

(e)

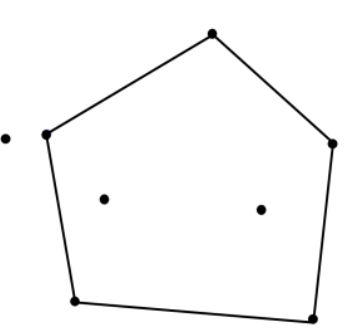

(b)

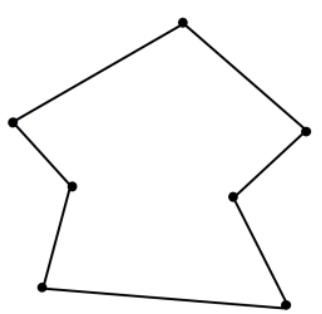

(d)

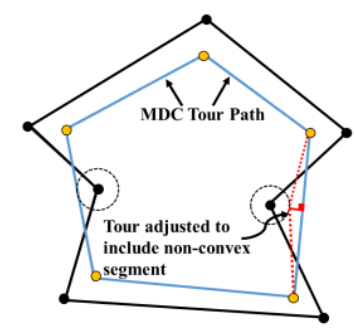

(f)
Figure 6: Illustrating the tour formation steps for a group of more than 2 terminals; (a) The layout of the terminals that need to be toured by an MDC; (b) The convex hull over the terminals; (c) Interior terminals find their nearest convex edge, (d) A simple polygon is formed by accounting for interior points; (e) Collection points are determined along angle bisectors for the convex vertices of the simple polygon forming a convex tour; (f) The tour is adjusted to account for collection points for any interior terminals. point is located along the angle bisector.

Proof: Consider Figure 7, which shows a part of the polygon with three terminals. Assume that our aim is to determine the collection point $P_{1}$ for terminal $S_{l}$, which will serve as a tour stop for a MDC. We know that $P_{l}$ has to be located in the interior of $S_{l}$, and can be at a maximum distance $R$, where $R$ is the communication range of a RN. Let the collection point $P_{1}$ lie along a line that divides the angle $\theta$ made by $S_{2}-S_{1}-S_{3}$ into $\theta_{1}$ and $\theta_{2}$, respectively. Since for the initial tour determination we consider only those terminals of a simple polygon, i.e., the vertices of the convex hull determined in Figure 6(b), we know that $\theta<\pi$ therefore $\theta_{1}<\pi$ and $\theta_{2}<\pi$. As seen in Figure 7 , the reduction in perimeter or tour length $\eta$ caused by having a collection point at $P_{l}$ can be approximated by:

$$
\eta=d_{1}+d_{2}, \quad \text { where } d_{1}=R \cos \theta_{1} \text { and } d_{2}=R \cos \theta_{2}
$$

Substituting in $d_{1}$ and $d_{2}$ we get

$$
\eta=R \cos \theta_{1}+R \cos \theta_{2}=R\left(\cos \theta_{1}+\cos \theta_{2}\right)
$$

Therefore for the reduction in perimeter to be maximal we need to maximize the value of $\left(\cos \theta_{1}+\cos \theta_{2}\right)$, which is equivalent to minimizing $\theta_{1}$ and $\theta_{2}$. Since $\theta=\theta_{1}+\theta_{2}$ we can replace $\theta_{2}=\theta-\theta_{1}$ and $\eta$ becomes:

$$
\eta=R\left(\cos \theta_{1}+\cos \left(\theta-\theta_{1}\right)\right)
$$

To maximize $\eta$ we differentiate both sides with respect to $\theta_{1}$.

$$
\frac{d \eta}{d \theta_{1}}=R\left((-1) \sin \theta_{1}+(-1)(-1) \sin \left(\theta-\theta_{1}\right)\right)
$$

The maximum $\theta_{1}$ corresponds to $\frac{d \eta}{d \theta_{1}}=0$, thus

$$
\begin{aligned}
& R\left(-\sin \theta_{1}+\sin \left(\theta-\theta_{1}\right)\right)=0 \\
& \Rightarrow \sin \theta_{1}=\sin \left(\theta-\theta_{1}\right) \\
& \quad \Rightarrow \theta_{1}=\theta-\theta_{1}
\end{aligned}
$$

Thus, $\theta_{1}=\frac{\theta}{2}$ and substituting in $\theta_{2}=\theta-\theta_{1}, \theta_{2}=\frac{\theta}{2}$. $\square$ 


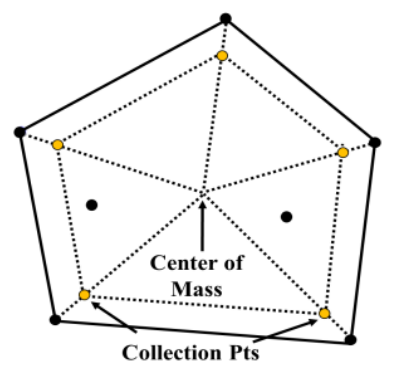

Figure 8: Illustrating the tour formation steps for competing approaches i.e. IDM-kMDC, MiMSI, MINDS. Basically, the collection points are determined along the line connecting the terminals to the center of mass.

Based on Theorem 3, having the collection point located on the angle bisector results in the maximum reduction in length, hence resulting in a smaller tour. So we determine the collection points for all terminals in the following manner. First we consider all the convex terminals in the simple polygon that we found in Figure 6(b) and determine their collection points by locating a point $R$ away along their angle bisectors towards the interior of the polygon. Joining these collection points gives us our initial convex tour which is nothing but the perimeter of the convex polygon as shown in Figure 6(e). If there are any interior terminals, we simply check their nearest convex tour edge and if it is within the transmission range, then the tour does not get adjusted since the MDC will pass in range of the said interior terminal. In case the terminal is more than $R$ units away from its nearest convex edge, the collection point is computed by: (i) finding the point of intersection of the perpendicular line from the terminal to the closest tour edge and its transmission disc, and then (ii) the tour edge is adjusted accordingly to account for the new collection point. This case is illustrated in Figure 6(f).

Figure 8 illustrates the tour formation in competing approaches such as MINDS [29], IDM-kMDC [30], and MiMSI [31]. They determine the tour by forming a convex hull of the terminals and then determine the center of mass. The point of intersection of the transmission disc of a terminal with a line connecting it to the center of mass serves as a collection point, i.e., the collection point is at a distance $R$ on the line between a terminal and center of mass as seen in Figure. A valid question would be why RCR ignores the center of mass and instead uses angle bisectors to determine the collection points. One reason is that by considering the convex hull, we ignore the interior terminals, and thus consider a much larger area than actually occupied by the terminals. Therefore the center of mass may conceivably be in an unfavorable position resulting in poor choices of collection points. Secondly Theorem 3 implies that populating along angle bisectors results in the largest reduction in perimeter. One can also ask why the collection points are not determined using the initial convex hull found in Figure 6(b) since the convex vertices of the simple polygon are utilized in determining the initial tour stops. The reason for obtaining the simple polygon is to minimize the angle $\theta$ at the convex vertices, thereby yielding further reduction in perimeter as compared to just using the convex hull and also helps in the final tour optimization phase, as will be explained in the next subsection.

Theorem 4: The runtime complexity of RCR tour formation

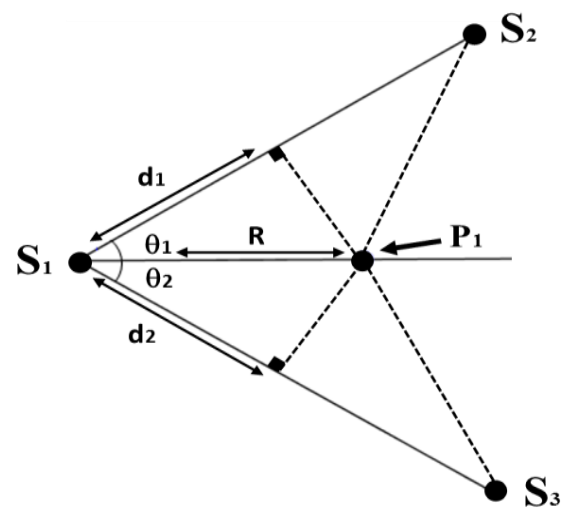

Figure 7: Determining the collection point of a MDC.

for a set of $n$ terminals is $O\left(n^{2}\right)$.

Proof: The convex hull over a set of $n$ points can be formed in $O(n \log n)$ using Graham scan. To form a simple polygon, each interior point needs to find its nearest convex edge. Say there are ' $k$ ' interior points so each point needs to check $(n-k)$ edges to determine the closest one, resulting in a time complexity of $O(k(n-k))$ or $O\left(n^{2}\right)$. The collection point can be determined in constant time for each vertex of a simple polygon giving a total time complexity of $O(n)$. So the overall time complexity of tour formation is $O\left(n^{2}\right)$.

\section{Tour optimization}

In this final phase, RCR aims to use any of the $l_{S}$ RNs that were unutilized in the segment grouping phase in order to further reduce the length of the MDC tours. A sorted queue in descending order of the MDC tour lengths is maintained and the available stationary RNs are then deployed to reduce the tour length of largest tour in the queue until all stationary RNs are utilized. Figure 9 is used in this subsection to illustrate the tour optimization for a MDC tour that spans seven terminals and has $l_{S}=3$. The objective of tour optimization is to equalize the travel load on the MDCs. Two main optimization steps are applies.

Optimization \#1: Generally, there are multiple positions where the RNs can be deployed. One option is to place a RN on a polygon edge between two terminals in order to shorten the distance between them; however such placement has no impact on the tour length since those terminals need to be

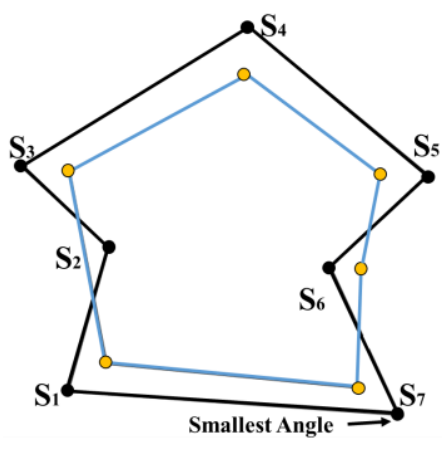

(a)

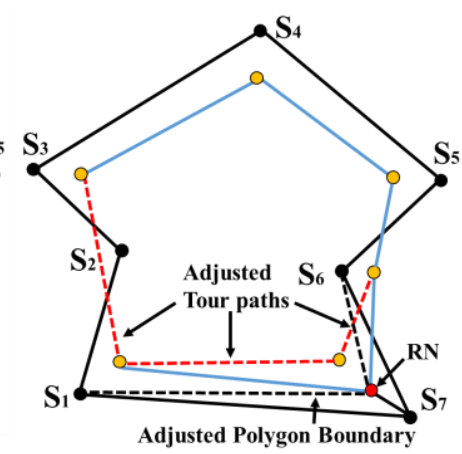

(b)

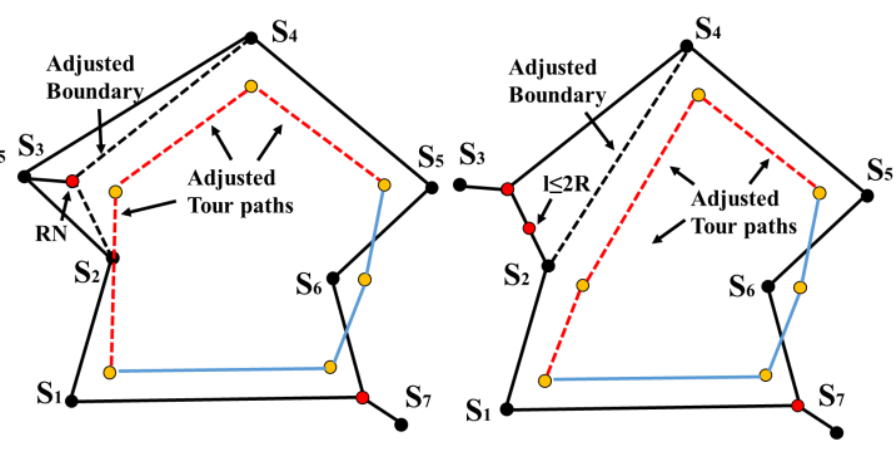

(c) (d)

Figure 9: Tour optimization of a MDC tour that spans seven terminals and $l_{S}=3$. (a) The smallest angle located at $\mathrm{S}_{7}$. (b) $\mathrm{RN}$ placed 'R' away along the angle bisector of $S_{7}$. (c) Next smallest angle located at $S_{3}$. (d) Tour optimization when a polygon edge has a length $\delta \leq 2 R$. 
visited regardless of the added RN. On the other hand, Theorem 3 implies that placing RNs along the angle bisectors results in the best choice to reduce the perimeter, so we can apply the same logic to reduce a MDC tour length.

Theorem 5: A reduction of approximately $2 \mathrm{R} \cos \frac{\theta}{2}$, in the perimeter of a polygon could be achieved, when an $\mathrm{RN}$ is placed along the angle bisector of the vertex (terminal) that has the smallest angle in the simple polygon.

Proof: Based on Theorem 3 the maximum reduction is achieved if a $\mathrm{RN}$ is placed at a distance $R$ from the terminal along the angle bisector. Using the equation of the perimeter in (1)

$$
\begin{aligned}
\eta & \approx R\left(\cos \theta_{1}+\cos \left(\theta-\theta_{1}\right)\right) \\
& \approx R\left(\cos \frac{\theta}{2}+\cos \frac{\theta}{2}\right) \\
& \approx 2 \mathrm{R} \cos \frac{\theta}{2}
\end{aligned}
$$

Therefore by having the smallest $\theta$ we can obtain the largest reduction which is nothing but choosing the smallest angle in the simple polygon. $\square$

As shown in Figure 9(b), the smallest angle is located at terminal $S_{7}$ and a RN is placed at its collection point to shorten the MDC tour. In the next iteration the smallest angle is at terminal $S_{3}$ as shown in Figure 9(c).

Optimization \#2: As argued above, placing an RN between two terminals connected by a polygon edge does not affect the overall MDC tour length; however, if we have a polygon edge that connects a convex terminal with an interior terminal of length $\delta \leq 2 R$, then by placing a RN on such an edge we can connect the 2 terminals. In other words, we can shrink the polygon by removing the convex terminal from consideration, and modifying its other incident edge to connect to the interior terminal. This leads to a smaller simple polygon and which in turn reduces the MDC tour. Figure 9(d) illustrates the idea through an example. In Figure 9(d), the length of the edge $S_{2} S_{3} \leq 2 R$, if we place an RN at the midpoint of $S_{2} S_{3}$, terminals $S_{2}$ and $S_{3}$ will be connected with one another and we can replace the edges $S_{3} S_{4}$ and $S_{2} S_{3}$ with the edge $S_{2} S_{4}$; thus we obtain a smaller simple polygon.

Theorem 6: If there exists a polygon edge with length $\delta \leq 2 \mathrm{R}$, that connects a convex terminal and an interior terminal, by placing a $\mathrm{RN}$ on that edge, a maximum perimeter reduction of 2R can be achieved.

Proof: Let us again consider Figure 9(d), particularly the edge $S_{2} S_{3}$. The reduction in the perimeter $\eta$ when applying the optimization to $S_{2} S_{3}$ is given by:

$$
\eta=S_{2} S_{3}+S_{3} S_{4}-S_{2} S_{4}
$$

Considering the triangle $S_{2} S_{3} S_{4}$, and applying the triangle inequality;

$$
S_{2} S_{4}+S_{2} S_{3}>S_{3} S_{4}
$$

Subtracting $S_{2} S_{4}$ from both sides we get,

$$
S_{2} S_{3}>S_{3} S_{4}-S_{2} S_{4},
$$

Note that $S_{2} S_{4}$ will always be longer than $S_{3} S_{4}$, since the interior terminal $S_{2}$ has to lie on the right of $S_{3}$ since RCR chooses the closest convex edge to $S_{3}$ when forming the simple polygon. Hence,

$$
\eta=S_{2} S_{3}-\Delta, \text { where } \Delta \text { is a positive number }
$$

Thus, we can conclude that $\eta \leq S_{2} S_{3} \leq 2 R$. $\square$

The removal of the edge $S_{2} S_{3}$ in Figure 9 (d) reduces the size of the simple polygon and thus yields a reduction in the MDC tour length as well. The removal of $S_{2} S_{3}$ also reduces the angle subtended at $S_{4}$ and further shrinks the tour length when the collection point is calculated. Therefore, by forming a simple polygon RCR simplifies the identification of edges $\leq 2 R$ in length, as only edges that are added when accounting for interior terminals are considered in this step.

In summary, RCR carries the tour optimization based on the following criteria; (1) In the simple polygon, find the terminal subtending the smallest angle and locate a point $R$ away on the angle bisector where a RN can be placed; (2) If there exists an edge with a convex and non-convex terminal as its endpoints and has an edge length $\leq 2 R$, place a $R N$ at the midpoint of the edge and remove the edge from the polygon. If both (1) and (2) are applicable at the same time RCR

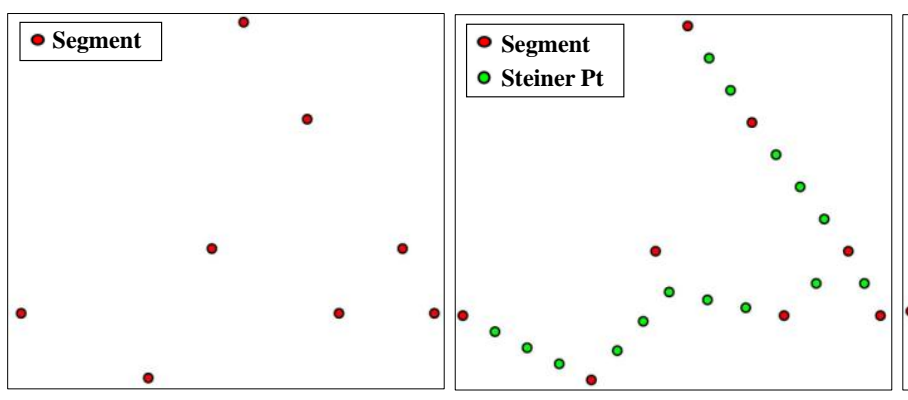

(a)

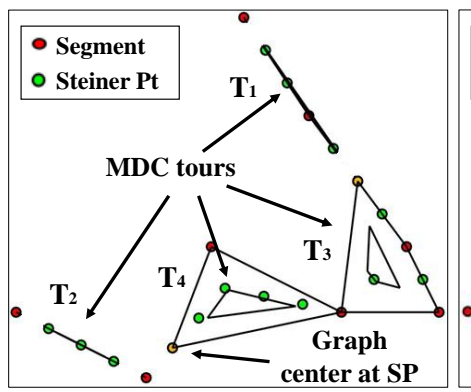

(e) (b)

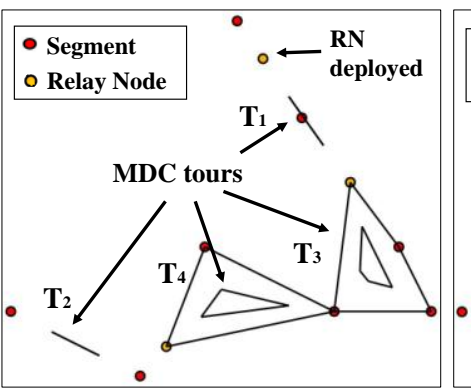

(f)

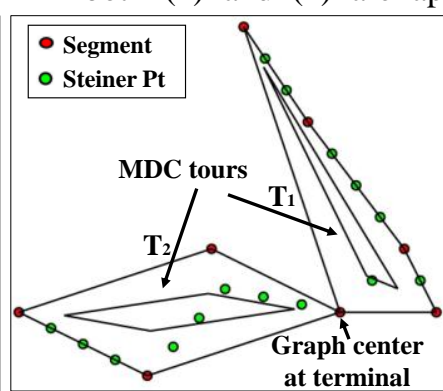

(c)

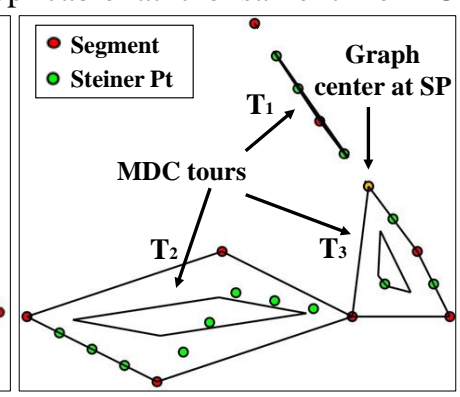

(d)

Figure 10: An illustrative example that demonstrates how RCR carries out segment grouping, tour formation and optimization when $1_{M}=4$ and $1_{S}=4$. (a) Initial set of eight terminals to be connected. (b) Since $1_{S}>1$, find Steiner points and determine the graph center (c) Divide mst into two groups around the graph center forming tours $T_{1}$ and $T_{2}$. (d) Partition the larger $T_{1}$, the graph center located at SP, so deploy a RN. (e) Divide the larger $\mathrm{T}_{2}$, the graph center located at SP, deploy a RN. (f) Number of Tours is equal to $1_{\mathrm{M}}$, number of available MDCs and $1_{\mathrm{S}}=2$. Tour optimization for largest tour $\mathrm{T}_{1}$ by deploying a RN. (g) Largest tour $\mathrm{T}_{4}$ optimized by placing a RN along the angle bisector of the smallest angle. (h) Final reconnected topology with 4 MDC tours. 
chooses the option that yields the most reduction in tour length for placing a $\mathrm{RN}$.

Theorem 7: The time complexity of tour optimization is $O\left(l_{S} n \log n\right)$, where $n$ is the number of terminals.

Proof: The smallest angle in the simple polygon can be found by only checking the ' $n$ ' convex vertices and sorting them in $O(n \log n)$. Finding edges of length $\leq 2 R$ between convex to non-convex vertices can be done in $O(k)$ time, where $k$ is the number of interior terminals. Since the addition of a RN affects only the edges incident on the vertex or edge being replaced, we do not need to recalculate the entire tour but simply replace the affected edges with new ones which can be done in constant time. The number of iterations for the tour optimization depends on the number of stationary RNs available which can at most be $l_{S}$. Therefore the time complexity of tour optimization is $O\left(l_{S} n \log n\right)$. $\square$

\section{E. Illustrative Example}

Figure 10 illustrates the application of RCR after the initial relay deployment phase is complete. Figure 10(a) shows eight terminals that need to be connected by utilizing 4 MDCs and 4 stationary RNs. RCR divides the segments into 4 groups one for each MDC. Since $l_{S}>1$, we form the SMT-MSPBEL utilizing IO-DT and incorporate the SPs found with the terminals in Figure 10(b).

We find the graph center over the set of terminals and SPs and split it into two tours $T_{1}$ and $T_{2}$ and keep recursively splitting the larger group until we obtain 4 groups, one for each MDC. In case the center falls on a SP we mark it as a location to populate a $\mathrm{RN}$ and decrement $l_{S}$ as shown in Figure 10(d) and 9(e). Once we have obtained the requisite number of groups we try to optimize the tours if we have any stationary RNs left. In this example we utilized two RNs during segment grouping and thus have two RNs available for tour optimization. The tours are maintained in a queue of descending order of their length, with the largest tour first. As indicated in Figure 10(e), $T_{l}$ is the largest tour and we employ a RN to shorten its length as seen in Figure 10(f). $T_{1}$ is reinserted it back in the tour queue. Now $T_{4}$ is the largest tour, RCR deploys the RN along the angle bisector of the smallest angle, as seen in Figure 10(g). Since there no more RNs left to deploy, RCR terminates. A pseudo code summary of RCR is provided in Figure 11.

\section{F. Overall Runtime Complexity}

Theorem 8: The time complexity of RCR is $O\left(\left(l_{M}-1\right) n^{2}\right)$.

Proof: In RCR, the grouping process runs at most $l_{M}-1$ times to obtain $l_{M}$ groups. The time to obtain the graph center is $O\left(n^{2}\right)$ by Lemma 3 and 4 . The tour formation also takes at most $O\left(n^{2}\right)$ as seen in Theorem 4 giving an overall time complexity of is $O\left((k-1) n^{2}\right)$. The tour optimization phase runs in $O\left(l_{S} n \log n\right)$. Hence the overall time complexity of $\mathrm{RCR}$ is $\left(l_{M}-1\right)\left(O\left(n^{2}\right)+O\left(n^{2}\right)\right)+O\left(l_{S} n \log n\right)$. Therefore the time complexity of RCR is $O\left(\left(l_{M}-1\right) n^{2}\right)$.

It is worth noting that the runtime complexity of IDMkMDC is $O\left(n^{4} \log n\right)$ [30], which is significantly higher than RCR. In comparison, in MiMSI the determination of SPs has a runtime complexity of $O\left(n^{4}\right)$ [10]. Kmeans++, the clustering algorithm used by MiMSI to form clusters has a runtime complexity of $2^{\Omega(\sqrt{n})}$ [34] where ' $n$ ' is the number of segments and SPs. Note that in MiMSI, due to the randomness in choosing cluster centers, if $l_{S}<l_{M}$ and all the cluster centers lie on SPs then merging needs to be carried out to meet the $1_{S}$ bound. The tour formation can be bounded by $O\left(n^{2}\right)$ and the optimization phase $O\left(l_{s} n \log n\right)$. Hence for MiMSI the runtime complexity can be bounded by $O\left(n^{4}\right)+2^{\Omega(\sqrt{n})}$, which is significantly more than RCR. In section $\mathrm{V}$, we compare the perfromance of the RCR to both IDM-kMDC and MiMSI through simulation.

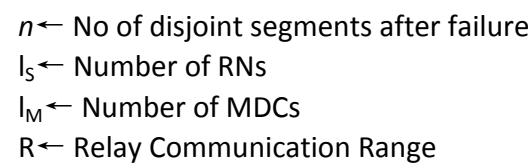

Initial Relay Deployment Phase [For every Seg $]$

-Identify Leading RN RN ${ }_{i 0}$ for $\mathrm{Seg}_{\mathrm{i}}$ and other spare $\mathrm{RNs}$ if available to follow in cascaded manner.

- Set $\mathrm{T}_{\text {Wait }}$ and wait until $\mathrm{T}_{\text {Wait }}=0$.

DO

IF disttoCenter $>\mathrm{R}$

Move RN towards Center O

IF meets $R N_{j k}$ or Seg shere $j \neq i$ THEN

Exchange Seg and RN information and STOP

ENDIF

ELSE

Become center RN and STOP

ENDIF

WHILE $\left(\mathrm{RN}_{\mathrm{i} 0} \neq \mathrm{STOP}\right)$

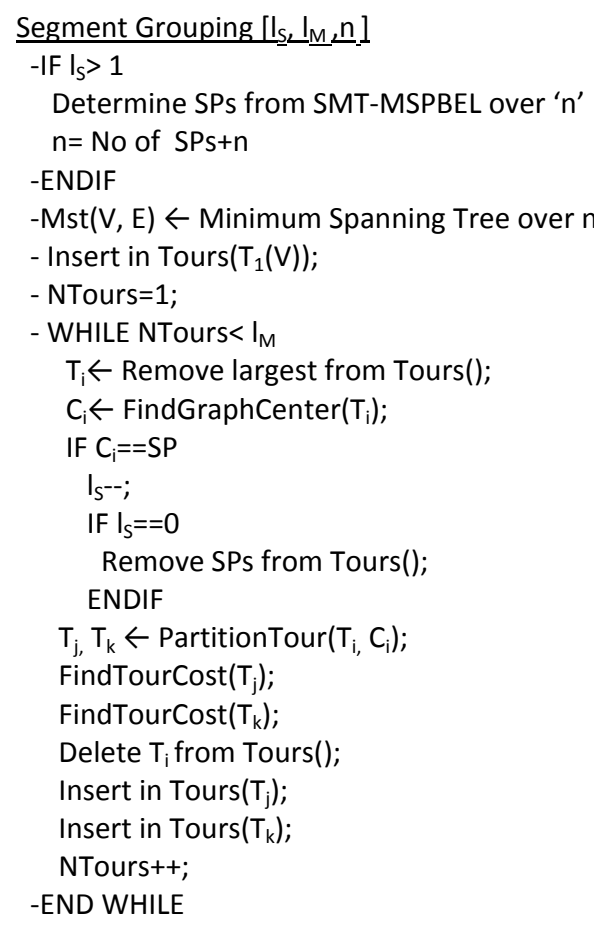

\section{Form Tours [Tours[]]}

-Form Convex Hull for all terminals in $T_{i}$

-Find Nearest edge to Interior pts and form Simple

Polygon.

-Calculate Collection points for all convex pts in $T_{i}$ along angle bisectors, forming initial tour.

-Determine collection points for non-convex terminals and adjust initial tour if necessary.

Tour Optimization [ Tour ]

WHILE $I_{S}>0$

Along largest tour deploy RN either along smallest angle or if edge length (Convex to non-convex terminal) $\leq 2 R$ and deploy along which ever gives maximum savings. 


\section{PERFORMANCE EVALUATION}

The effectiveness of RCR is validated through simulation. This section discusses the simulation setup, performance metrics and results.

\section{A. Performance Metrics and Experiment Setup}

The experiments are conducted in a $1500 \times 1500 \mathrm{~m}^{2}$ area wherein random topologies are generated for a varying number of segments ( 5 to 25 ) and communication range $\mathrm{R}$ is fixed at $100 \mathrm{~m}$ for all RNs. The CGAL computation library [36] was used to carry out all geometric computations. We consider the following metrics to measure performance:

- Total Tour length: is the sum of tour lengths of all RNs employed as MDCs by RCR. A smaller total tour length implies lower overhead on the MDCs.

- Maximum Tour length: is the length of the longest tour amongst all MDC tours. A large value implies higher travel overhead on a MDC and increased data latency.

- Standard Deviation: measures the dispersal of tour lengths from the average tour length. A low value indicates that the tour lengths are very close to the average and the travel load is being shared equitably amongst the MDCs. A large value indicates unbalanced tours, which can result in poor data latency and cause losses due to buffer overflows in segments being covered by the longest tour. An overloaded MDC is also prone to running out of battery life quicker.

We study the performance while varying the following parameters:

- Ratio of available RNs ( $\Phi)$ : Since RCR aims to solve the recovery problem when the surviving segments do not have enough RNs to form a stable inter-segment topology, we use $\Phi$ to control the RN count. The total number of available RNs is set to the number of Steiner points found in the
SMT-MSPBEL multiplied by $\Phi$, whose value is set at 0.45 and 0.55 respectively. Out of the available RNs, the ratio of $l_{M}$ mobile to $l_{S}$ stationary RNs is fixed at 1 , i.e., $l_{M} / l_{S}=1$.

- Number of Segments $\left(N_{\text {seg }}\right)$ : A higher number of disjoint segments in a WSN introduces more connectivity requirements between them and highlights the complexities of tour formation.

\section{B. Simulation Results}

This section discusses the obtained results. Each configuration is averaged over 50 random topologies. We observed that with a $90 \%$ confidence interval, the results stayed within 5\%-10\% of the sample mean.

In the first set of experiments we compare RCR with MiMSI [31], which also tackles the connectivity restoration problem using a mix of MDCs and stationary RNs. In these experiments the communication range of the RNs is fixed at $100 \mathrm{~m}$. The graphs in Figure 12 and 13 show the variation in the total distance travelled by all MDCs, the maximum overhead on a MDC, and the standard deviation of the MDC tours as the number of segments are varied from 5 to 15 . We also vary $\Phi$, to highlight the effect increased resources have on connectivity restoration. Figure 12 (a) and 13(a) show that RCR has a lower combined total tour length than MiMSI. This improvement is due to better segment grouping, which results in balanced tours and also by having a superior tour formation strategy which reduces the MDC travel overhead. We also see that as $\Phi$ increases the performance advantage for RCR grows larger, this is because larger $\Phi$ implies more MDCs and stationary RNs are available, thus more tours are formed and the advantages of RCR tour formation add up, amplifying the saving and resulting in a larger gap with respect to MiMSI. We see that the gap in total tour length decreases with the increase in the number of segments because the inter-segment

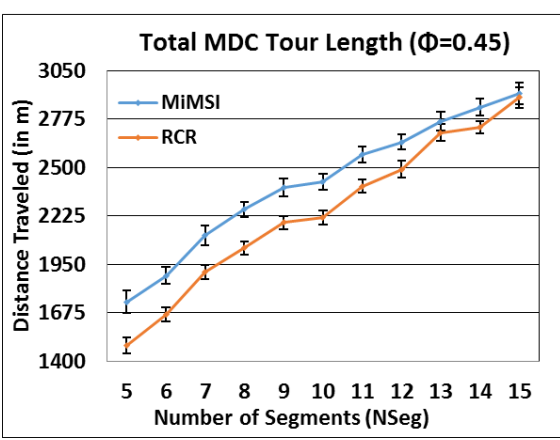

(a)

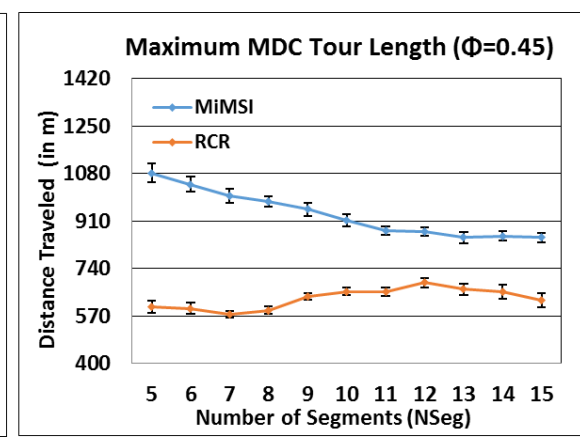

(b)

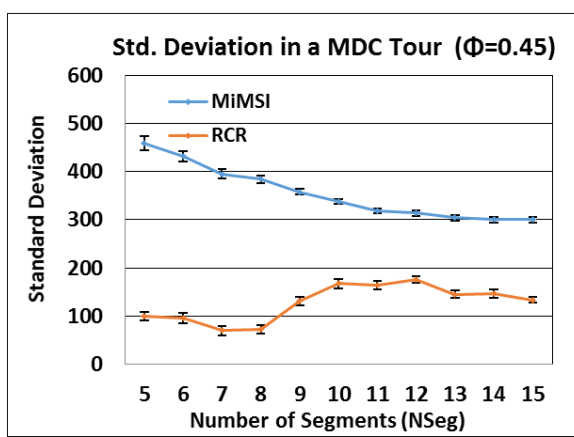

(c)

Figure 12: Total distance Traveled by MDCs for $\Phi=0.45$ and communication range $R=100 \mathrm{~m}$.

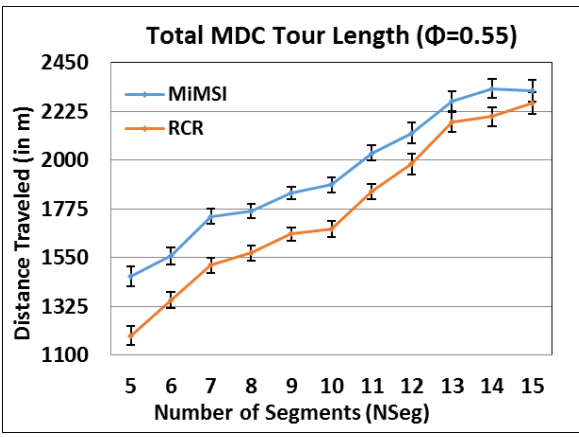

(a)

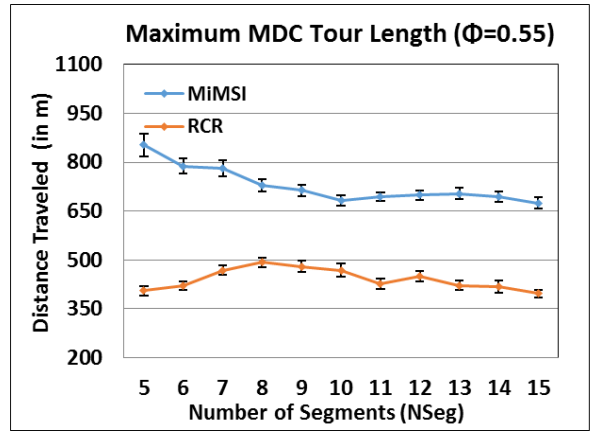

(b)

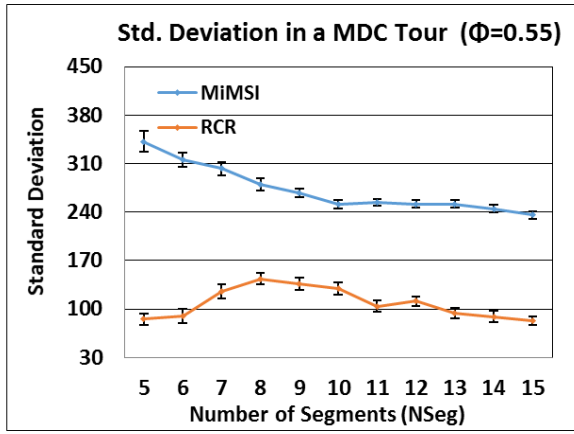

(c)

Figure 13: Total distance Traveled by MDCs for $\Phi=0.55$ and communication range $R=100 \mathrm{~m}$. 
proximity increases and the drawback of MiMSI's clustering algorithm diminishes. Recall that unlike RCR, the kmeans++ algorithm used by MiMSI does not factor inter-cluster distance. Thus, the increased segment density slighly improves the clustering performance in MiMSI.

Figure 12(b) and 13(b) show that RCR handily outperforms MiMSI by having a much lower maximum tour load on a MDC, while Figures 12(c) and 13(c) indicate that the standard deviation is also much lower for RCR generated MDC tours. A lower standard deviation in tour length is beneficial because it means that the tours are approximately equal in size. Having balanced tours means that no one MDC is overburdened into covering a larger area, which can make it run out of energy quicker than other MDCs. Obviously, losing an MDC due to energy exhaustion would mean re-calculating MDC tours again which is an additional overhead.

As $\Phi$ is varied from 0.45 to 0.55 we see a reduction in the total tour length and maximum tour length (Figures 12 and 13). This is because an increase in $\Phi$ means availability of more resources, i.e., MDCs and stationary RNs to cover the same WSN. Increased RN availability means that the segment groups will be smaller since more MDCs are available and the extra stationary RNs can be utilized in the tour optimization phase to reduce the longest MDC tours.

To show that our segment grouping technique is superior to that of MiMSI, we run a set of experiments where we compare two versions of RCR, one utilizing kmeans++ clustering, like MiMSI, and the other applies our segment grouping technique. The tour optimization and formation procedure is kept the same in both cases. The results in Figure 14(a) and 14(b) show a reduction in total tour length for the kmeans++ based RCR compared to MiMSI; such a reduction is attributed to our tour formation technique. However, from Figure 14 and 15 it is clear that RCR's graph center based segment grouping is superior in all metrics like the total tour length, maximum overhead on a MDC and standard deviation amongst MDC tours. In addition to providing better results it is also much faster than kmeans++ which has superpolynomial time complexity, as we discussed in Section IV.

Finally, we compare the performance of RCR with recovery solutions that deal with the general case in which all available RNs can be utilized as MDCs. For this comparison

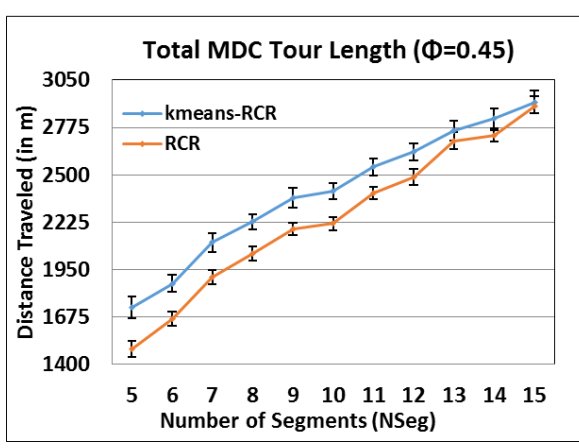

(a)

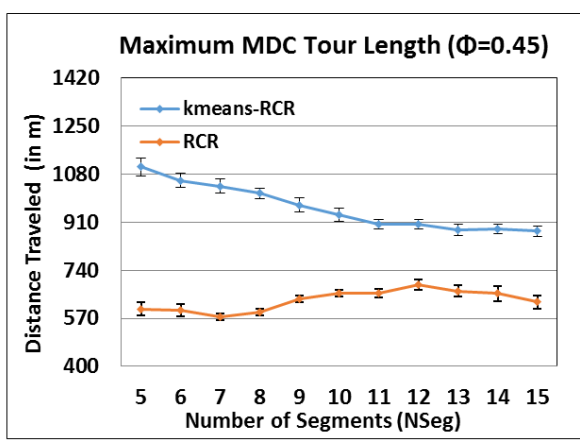

(b)

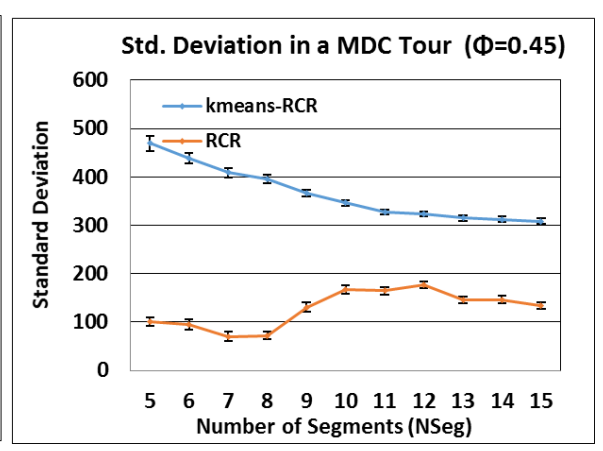

(c)

Figure 14: Comparing the clustering method of RCR with MiMSI for $\Phi=0.45$ and communication range $\mathrm{R}=100 \mathrm{~m}$.

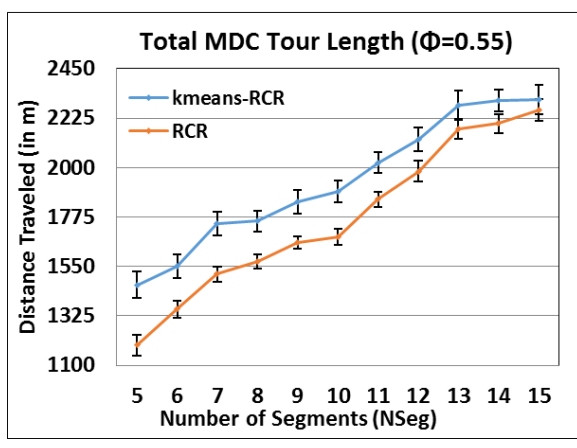

(a)

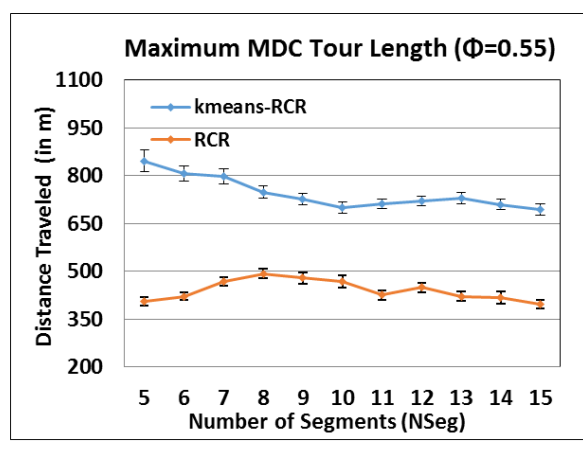

(b)

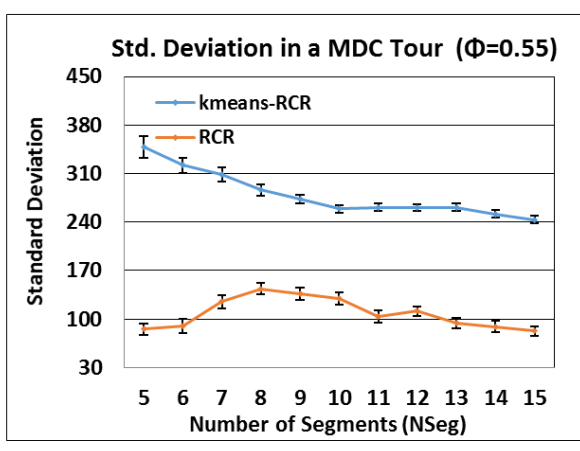

(c)

Figure 15: Comparing the clustering method of RCR with MiMSI for $\Phi=0.55$ and communication range $\mathrm{R}=100 \mathrm{~m}$.

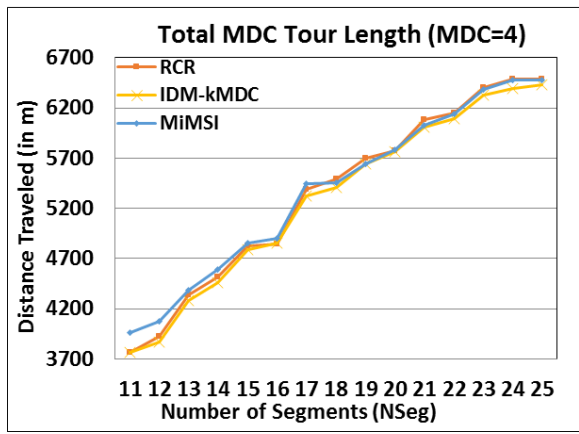

(a)

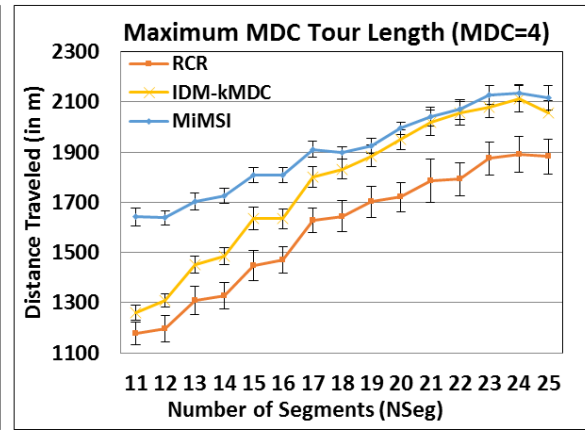

(b)

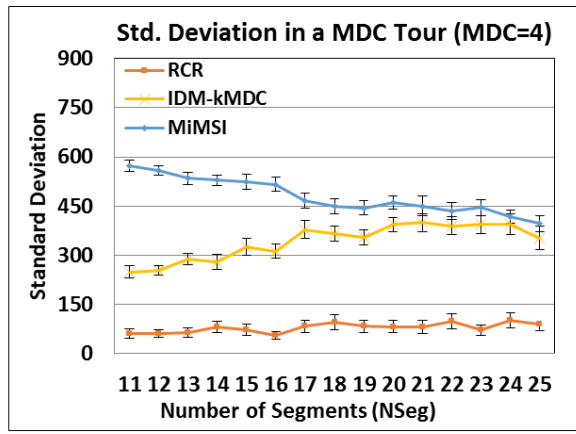

(c)

Figure 16: Comparison of RCR with IDM-kMDC and MiMSI for fixed MDC count, namely, $l_{M}=4$ and $l_{S}=0$. 
we choose IDM-kMDC, the best performing approach that aims to minimize the overall MDC tour length. IDM-kMDC is a greedy approach, that forms an mst over the set of disjoint segments and assigns an MDC to each mst edge, and keeps combining tours that have the least merging cost until the number of tours meets the MDC availability constraint. Since IDM-kMDC considers all RNs to be mobile, to keep the comparison consistent we have set the number of stationary RNs to zero $\left(l_{S}=0\right)$ and run the experiment for a fixed number of $\operatorname{MDC}\left(l_{M}=4\right)$ while varying the number of segments between 11 and 25. The number of MDCs is kept less than the number of segments in order to engage the tour formation algorithm of IDM-kMDC. The communication range $\mathrm{R}$ is fixed at $100 \mathrm{~m}$. Figure 16(a) shows that all approaches have approximately the same total tour length. Figure 16(b) and 16(c) clearly highlight that RCR forms more balanced tours, while having approximately the same total tour length as the competing approaches. RCR not only has the smallest maximum tour length amongst all three approaches but the standard deviation of the tours formed is also the lowest. This means that in RCR MDCs have a balanced work load and the data delivery latency will be much less in comparison with IDM-kMDC and MiMSI.

Overall, the simulation results in Figures 12-16 confirm the performance advantage of RCR. RCR restores intermittent connectivity in a resource-constrained WSN and is shown to impose significantly less overhead on MDCs than competing approaches by utilizing a lightweight and effective segment grouping approach that produces balanced MDC tours. Also by exploiting the geometric properties of angle bisectors and polygons, RCR produces smaller tours over the same set of terminals as compared to other approaches. RCR segment grouping and tour formation reduce the total distance that MDCs have to travel for data exchange which translates into savings in energy and also improved the network lifetime. The performance advantage of RCR grows significantly as the percentage of RNs available grows since it means more MDCs are available and more tours are formed, thus increasing the gain in performance due to better segment grouping and tour formation.

\section{CONCLUSION}

An autonomous WSN should be able to recover from a large scale failure that damages multiple collocated nodes. In practical scenarios based on the level of damage the surviving segments in the network may not have enough resources to form a stable inter-segment topology. In this paper we have presented RCR, a novel distributed algorithm for restoring connectivity in a resource constrained WSN. RCR takes into account that not all available RNs can function as MDCs and some may have to be stationary due to low remaining energy or some external damage that impedes their movement. RCR achieves the recovery goal by initially relocating the fewest RNs from individual segments and then plans MDC tours and placement of stationary RNs to facilitate inter-segment connectivity. The simulation results have demonstrated that RCR scales well and outperforms competing approaches.

ACKNOWLEDGEMENTS: This work was supported by the National Science Foundation (NSF) award \# CNS 1018171.

\section{REFERENCES}

[1] M. Younis, I. Senturk, K. Akkaya, S. Lee and F. Senel, "Topology Management Techniques for Tolerating Node Failures in Wireless Sensor Networks: A Survey," Computer Networks, Vol. 58, pp. 254-283, Jan 2014.

[2] X. Han, X. Cao, E. L. Lloyd and C.-C. Shen, "Fault-tolerant Relay Nodes Placement in Heterogeneous Wireless Sensor Networks," Proc. of INFOCOM'07, Anchorage AK, May 2007.

[3] N. Li and J. C. Hou, "Flss: a fault-tolerant topology control algorithm for wireless networks," Proc. of MobiCom '04, Philadelphia, PA, Sept 2004.

[4] X Wang, G Xing, Y Zhang, C Lu, R Pless and C Gill, "Integrated coverage and connectivity configuration in wireless sensor networks", Proc. of ACM Sensys'03, Los Angeles, CA, November 2003.

[5] B. Chen, et al., "Span an energy-efficient coordination algorithm for topology maintenance in ad hoc wireless networks," Proc. of ACM MobiCom'01, Rome, Italy, July 2001.

[6] G. Wang, G. Cao, T. La Porta, and W. Zhang, "Sensor Relocation in Mobile Sensor Networks," Proc. INFOCOM'05, Miami, FL, Mar. 2005.

[7] G. Lin, G. Xue, "Steiner Tree Problem with Minimum Number of Steiner Points and Bounded Edge-length," Information Processing Letters, 69(2), pp. 53-57, 1999.

[8] X. Cheng, D.-z. Du and L. Wang and B. Xu, "Relay Sensor Placement in Wireless Sensor Networks," Wireless Networks, 14(3), pp. 347-355, 2008.

[9] E. L. Lloyd, G. Xue, "Relay Node Placement in Wireless Sensor Networks," IEEE Trans. on Comp., 56(1), pp. 134-138, Jan 2007.

[10]F. Senel, M. Younis, Relay node placement in structurally damaged wireless sensor networks via triangular steiner tree approximation, Elsevier Computer Communications 34 (16) (2011) 1932-1941.

[11] F. Senel and M. Younis," Optimized Relay Node Placement for Establishing Connectivity in Sensor Networks" Proc. of Globecom'12, Anaheim, CA, Dec. 2012.

[12] Y. K. Joshi and M. Younis, "Autonomous Recovery from Multi-node Failure in Wireless Sensor Network" Proc. of Globecom'12, Anaheim, CA, Dec. 2012.

[13] S. Lee, and M. Younis, "Recovery from Multiple Simultaneous Failures in Wireless Sensor Networks using Minimum Steiner Tree," Journal of Parallel and Distributed Computing, Vol. 70, pp. 525-536, April 2010.

[14]Y. K. Joshi and M. Younis, "Distributed Approach for Reconnecting Disjoint Segments” Proc. of Globecom'13, Atlanta, GA, Dec. 2013.

[15]Y. K. Joshi and M. Younis, "Straight Skeleton Based Reconnection in a Wireless Sensor Network” Proc. of Globecom'14, Austin, TX, Dec. 2014.

[16]Y.K. Joshi, M. Younis, Exploiting skeletonization to restore connectivity in a wireless sensor network, Computer Communications (2015).

[17]S. Das et al., Localized movement control for fault tolerance of mobile robot networks, in: Proc. of the 1st IFIP Int'l Conference on Wireless Sensor and Actor Networks (WSAN 2007), Albacete, Spain, Sept. 2007.

[18]K. Akkaya, F. Senel, A. Thimmapuram, S. Uludag, "Distributed Recovery from Network Partitioning in Movable Sensor/Actor Networks via Controlled Mobility," IEEE Trans. on Comp., 59(2), pp.258-271, 2010.

[19] F. Senel, K. Akkaya and M. Younis, "An Efficient Mechanism for Establishing Connectivity in Wireless Sensor and Actor Networks," Proc. of Globecom'07, Washington, DC, Nov. 2007.

[20]R. Shah, S. Roy, S. Jain, W. Brunette, "Data MULEs: modeling a three tier architecture for sparse sensor networks", in: Proc. of IEEE Int' Workshop on Sensor Network Protocols and Applications (SNPA'03), May 2003.

[21]S. Jain, R.C. Shah, W. Brunette, G. Borriello, S. Roy, "Exploiting mobility for energy efficient data collection in wireless sensor networks", Journal of Mobile Networks and Applications, Vol. 11, pp. 327-339, June 2006.

[22]W. Alsalih, S.G. Akl, H.S. Hassanein, "Placement of multiple mobile base stations in wireless sensor networks" Proc. of ISSPIT'07, Cairo, Egypt, Dec 2007.

[23]W. Alsalih, H. Hassanein, S. Akl, "Placement of multiple mobile data collectors in wireless sensor networks", Journal of Ad Hoc Networks, Vol. 8, pp. 378-390, June 2010.

[24]H. Almasaeid, A.E. Kamal, "Data delivery in fragmented wireless sensor netowrks using mobile agents" Proc. of MSWiM 07, Chania, Greece, Oct 2007.

[25]C.-C. Shen, O.Koc, C. Jaikaeo, Z. Huang, "Trajectory control of mobile access points in MANET" Proc. of Globecom'05, St. Louis, MO, Nov.2005.

[26]G. Robins, A. Zelikovsky, "Tighter bounds for graph steiner tree approximation," SIAM J. on Discrete Math., 19(1), pp.122-134, 2005.

[27]S. Vemulapalli and K. Akkaya, "Mobility-based Self Route Recovery from Multiple Node Failures in Mobile Sensor Networks", Proc. of WLN '10, Denver, CO, Oct. 2010. 
[28] J. Munkres "Algorithms for the Assignment and Transportation Problems" J. Society for Industrial and Applied Math. 5(1), Mar. 1957

[29]Y. K. Joshi and M. Younis, "Mobility-based internetworking of disjoint segments," Proc. of $27^{\text {th }}$ Biennial Symposium on Communications (QBSC), Kingston, Ontario, Canada, June 2014.

[30]F.Senel, M. Younis, "Optimized Interconnection of Disjoint Wireless Sensor Network Segments Using K Mobile Data Collectors," Proc. of Int'l Conf. on Comm. (ICC'12), Ottawa, Canada, Jun 2012.

[31]A. Abbas and Mohamed Younis. "Establishing connectivity among disjoint terminals using a mix of stationary and mobile relays," Computer Communications Vol 36, No 13, pp. 1411-1421, June 2013.

[32] D. Arthur, S. Vassilvitskii, "k-means++: the advantages of carefu seeding," Proc. of the 18th Annual ACM-SIAM Symposium on Discrete Algorithms (SODA'07), Philadelphia, PA, January 2007.
[33]D.L. Applegate, R.E. Bixby, V. Chvátal, W. J Cook, "The Traveling Salesman Problem: A Computational Study," Princeton University Press, New Jersey, USA, 2006.

[34]D. Arthur and S. Vassilvitskii, "How slow is the k-means method?" Proc of SCG '06, Sedona, AZ, June 2006.

[35]S. Wuchty and Peter F. Stadler. "Centers of complex networks," Journal of Theoretical Biology Vol 223, No 1, pp. 45-53 July 2003.

[36]CGAL, Computational Geometry Algorithms Library, http://www.cgal.org 